\begin{tabular}{|l|l|l|l|}
\hline Eiszeitalter u. Gegenwart & 33 & $\begin{array}{l}21-30 \\
4 \mathrm{Abb} .\end{array}$ & Hannover 1983 \\
\hline
\end{tabular}

\title{
Terrassenverstellungen und tektonische Analyse von Satellitenbildern - ein methodischer Versuch, dargestellt an Beispielen aus dem Mosel-Saar-Nahe-Raum
}

\author{
YOUSRY WAHBA \& LUDWIG ZÖLleR *)
}

Morphogenesis, fluvial terrace, alluvion, tectonic analysis, displacement, Cenozoic, systematic approach, landsat.

Rhenish Schiefergebirge, Nahe River, Monzingen Nußbaum Area, Simmerbach Idar-Oberstein Area, Saar Nahe Basin, Saar River, Können, Rhineland Palatinate.

TK25: Nr. 6205, 6209, 6210, 6211

$\mathrm{K}$ u r z f a ssung: Angesichts der Schwierigkeit, känozoisch aktive Störungen oder Flexuren in gehobenen Gebieten mit nur lückenhaft erhaltenen känozoischen Deckschichten (z. B. als Terrassen) nachzuweisen, wurde versucht, fotogeologische Methoden als Hilfsmittel einzusetzen.

Von Landsat-I- und II-Aufnahmen wurde das Bruchmuster der betreffenden Region auskartiert. Das Fotolineationenmuster wurde in zwei Arbeitsschritten eingesetzt:

1. Lineationen wurden in die Feldkarten übertragen, um die Geländearbeiten auf die unmittelbare Umgebung der Lineationen zu konzentrieren, wo Höhenunterschiede an der Oberfläche und an der Basis känozoischer Ablagerungen untersucht wurden.

2. Anhand der fotogeologisch erkannten Bruchstrukturen wurden Großschollen mit typischem

Richtungsverteilungsmuster gegeneinander abgegrenzt. Sodann wurde untersucht, ob Terrassenlängsprofile an den Großschollengrenzen signifikante Gefällsbrüche aufweisen.

Im Zuge einer kritischen Beurteilung der Ergebnisse werden der Abbildungscharakter von Fotolineationen, die tektonische Untersuchung nicht verfestigter Sedimente und die Flußmorphologie diskutiert, sofern sie für diese Arbeit von Bedeutung sind.

An einigen Beispielen aus den Talregionen von Mosel, Saar, Prims und Nahe wird gezeigt, daß die Methode erfolgversprechend angewendet werden kann: in einigen Fällen bilden Lineationen in Terrassenkörpern mit hoher Wahrscheinlichkeit junge Störungen von zweitrangiger Bedeutung ab.

Zonen mit stärker gehobenen Terrassen oder Tertiärsedimenten zeichnen sich durch ein charakteristisches Muster von Lineationen $a b$ und werden von langgestreckten Lineamenten begrenzt, die möglicherweise jungen Flexuren folgen.

\section{[Displacement of Fluvial Terraces and Tectonic Analysis of Satellite Imagery - a Methodological Attempt, Illustrated by Examples from the Mosel-Saar-Nahe-Region]}

Abstract: Facing the problem that cainozoic activities of block faults or flexures can hardly be proved in uplifted regions without a coherent cover of cainozoic sediments, where only relics of them have been preserved (for example as river terraces), we tried to use photo-geological methods as auxiliary means. LANDSAT I and II-scenery was analysed to find out the block fault patterns of the regions under consideration.

We operated in two steps: First, lineations were transscribed into the field map to concentrate field work to the localities close to the lineations in order to recognize height differences of the surface and the bottom of cainozoic sediments.

Secondly, the photo-geological block fault pattern was analyzed, and the considered regions were divided into superimposed block units with characteristic directions of lineations. The classification of units was compared with significant discontinuities of longitudinal sections of river terraces.

Problems concerning the character of lineations, tectonic investigation of non consolidated sediments, and fluvial morphology are discussed as far as they are of interest for our investigation.

*) Anschrift der Autoren: Dipl.-Geologe Dr. Y. W a h b a, Brunnenstraße 42, 5501 Gusterath, W'-Germany. - L. Z ö 11 e r, M. A., Kirchstraße 16, 5501 Thomm, W-Germany. 
Some examples taken from the valleys of the rivers Moselle, Saar, Prims, and Nahe illustrate that our method can be applied successfully: in some cases it was plausible that lineations traversing river terraces describe block faults of secondary importance.

Zones of stronger uplifted river terraces or tertiary sediments can be identified by their characteristic mosaic of photolineations and are bordered by extended lineations, which may describe fiexures.

\section{Problem}

Terrassenverstellungen und -verbiegungen sind im Rheinischen Schiefergebirge bzw. an seinen Rändern seit langem bekannt und vielfach untersucht worden. Während in Beckenregionen wie z. B. der Niederrheinischen Bucht mit weiträumiger Erhaltung pleistozäner fluviatiler Sedimente Störungen in den Terrassenkörpern unmittelbar nachweisbar sind (AHORNER 1962; BoENigK 1978), ist man in zentralen Teilen des Gebirges meistens auf eine mehr oder weniger hypothetische Parallelisierung von Terrassenrelikten über Schollengrenzen hinweg angewiesen, um tektonische Verstellungen zu erkennen. Die junge Kerberosion hat in Hebungsgebieten bevorzugt den Störungen und Kluftzonen - auch den quartären - nachgetastet und folglich gerade an den Stellen, wo eine junge Störung $\mathrm{zu}$ vermuten ist, die Terrassen vielfach abgetragen. Eine weitere Schwierigkeit besteht darin, daß Störungen im Liegenden selbst mit Beträgen im Meter- oder Dekameterbereich oft wegen der Uniformität der mächtigen devonischen oder rotliegenden Gesteinsserien kartiertechnisch kaum zu erfassen sind. Schließlich erschwert die Möglichkeit, daß Terrassenverstellungen an einer Flexur oder an einer Vielzahl parallelverlaufender kleiner Brüche erfolgt sein können, ihren Nachweis im Gelände, da Höhenunterschiede von Terrassenunter- und -oberkanten bis zu einigen Metern auch nichttektonisch erklärt werden können.

Angesichts dieser Schwierigkeiten wurde versucht, die durch die moderne Fotogeologie bereitgestellten Methoden zur Erfassung des tektonischen Inventars eines Raumes (vgl. Kronberg 1977) für die Untersuchung der quartären Tektonik nutzbar zu machen.

\section{Methodik}

Von dem zu untersuchenden Raum - dem Moselgebiet zwischen Trier und Koblenz, dem Saartal und dem Saar-Ruwer-Hunsrück, dem Primsgebiet und dem Nahegebiet einschließlich des Hunsrücksüdrandes von Bad Kreuznach an flußaufwärts - wurden LANDSAT-I- und II-Aufnahmen in Schwarz-Weiß auf ca. $1: 200000$ vergrößert und Fotolineationen anhand von Grautonunterschieden nach der in KRONBERG (1977) beschriebenen Methode auskartiert. Die unmittelbare Übertragung der Lineationen auf die Topographische Karte 1:200000 ermöglichte die Eliminierung von Eisenbahnlinien, größeren gradlinigen Straßenabschnitten etc. Da die Parzellengrößen im untersuchten Raum im allgemeinen klein und die Fluren stark zerstückelt sind, wirken sich Feldergrenzen bei diesem Abbildungsmaßstab allgemein nicht störend aus. Allenfalls größere geradlinige Waldgrenzen können als nicht-tektonische Lineationen kartiert werden, sie folgen aber meistens Gesteinsgrenzen (z. B. Quarzite/Schiefer im Hunsrück).

Die Fotolineationen-Karten fanden in zweifacher Hinsicht Anwendung:

1. die Lineationen wurden in die Feldkarten $1: 25000$ übertragen. Dieser Maßstab entspricht zwar vielfach nicht mehr dem Auflösungsvermögen der LANDSAT-Aufnahmen (nach Kronberg 1977 beträgt es 80-100 m); diesen Mangel konnte aber die Erfahrung, daß Fotolineationen häufig markanten Reliefeinheiten wie Kerbtälchen, Geländerissen und Schluchten, Steilhängen etc. folgen, weitgehend ausgleichen. Die Terrassen wurden an Stellen, wo sie von Lineationen durchzogen werden, besonders genau darauf untersucht, ob an der Oberfläche kleine Kanten bzw. Stufen aufzufinden sind, oder ob eine Verstellung der 
Terrassenbasis erkennbar ist. Die im Gelände gewonnenen Daten wurden durch die Auswertung zahlreicher Trinkwasser- und Aufschlußbohrungen ergänzt.

2. Im größeren räumlichen Kontext wurde versucht, Schollen mit einem typischen tektonischen Muster (Bruchmuster), d. h. Schollen mit einer Vergitterung charakteristischer Richtungen von Lineationen, die durch langgestreckte Lineamente voneinander getrennt werden, abzugrenzen. Die so ausgewiesene Schollengliederung wurde zu den Terrassenlängsprofilen in Beziehung gesetzt, um zu überprüfen, ob Terrassenverstellungen mit fotogeologischen Schollengrenzen zusammenfallen. Lassen sich derartige Kongruenzen feststellen, kann die fotogeologische Schollengliederung als Hilfsmittel zur flächenhaften Abgrenzung von Hebungs- oder Absenkungsgebieten eingesetzt werden, während durch Terrassenlängsprofile alleine nur eine eindimensionale Erfassung der posthumen tektonischen Bewegungen gelingt.

\section{Resultate}

Nur bei einem Bruchteil der auskartierten Lineationen konnten känozoische tektonische Bewegungen im Gelände nachgewiesen oder wahrscheinlich gemacht werden. Lineationen, die Terrassenkörper durchsetzen, konnten in einigen Fällen als Störungen gedeutet werden, mit unterschiedlicher Häufigkeit in einzelnen Regionen. An einer Vielzahl derartiger Lineationen war aber keine Verstellung der Terrassenbasis zu erkennen, so daß vorläufig offen bleiben muß, ob sie überhaupt (durchgepauste) tektonische Trennflächen abbilden. Allerdings muß berücksichtigt werden, daß Horizontalverschiebungen in Terrassenkörpern im Gelände noch viel schlechter nachweisbar sind als Störungen mit Vertikalversatz, da selbst in Aufschlüssen wegen der lockeren Lagerung der Sedimente Horizontalbewegungen kaum erkennbar sind und die Terrassenbasis als Bezugshorizont ausscheidet.

Eine weitere Schwierigkeit muß bei der vergleichenden Interpretation der Terrassenlängsprofile und der Fotolineationen unbedingt beachtet werden: die Basisfläche fluviatiler Ablagerungen ist selten vollständig eben. Rinnen können wenige Meter tiefer ins Anstehende eingeschnitten sein als die durchschnittliche Höhenlage der Auflagerungsfläche, die zudem meistens zum (damaligen) Stromstrich geneigt ist, so daß Höhenunterschiede bis zur Größenordnung der Mächtigkeit der Terrassensedimente auch nicht-tektonisch erklärt werden können (an der Mosel 5-10 m, sonst selten über $5 \mathrm{~m}$ ).

Trotzdem können wir einige Beispiele aufführen, in denen Fotolineationen in oder zwischen Terrassenkörpern mit hoher Wahrscheinlichkeit posthum aktive Störungen abbilden.

\subsection{Moselgebiet (Y. WAHBA) (Abb. 1)}

In der geologisch-tektonischen Karte zeichnen sich die einzelnen Störungssysteme ganz deutlich als Teilstück des jeweiligen Richtungstrends der regionalen Bruchtektonik des Gebietes $\mathrm{ab}$.

\begin{tabular}{l|c|l} 
Großscholle & $\begin{array}{l}\text { Streichrichtung der } \\
\text { Lineationen innerhalb } \\
\text { der Großscholle }\end{array}$ & $\begin{array}{l}\text { Lineationen zwischen Groß- } \\
\text { schollen und Streichrichtung }\end{array}$ \\
\hline A. Trier-Brauneberg & $5-20^{\circ} \mathrm{NNE}-\mathrm{SSW}$ & Liesertal-Lineation $150^{\circ} \mathrm{NW}-\mathrm{SE}$ \\
B. Brauneberg-Mont Royal & $8^{\circ} \mathrm{N}-\mathrm{S}$ & Alf-Lineation $150^{\circ} \mathrm{NW}-\mathrm{SE}$ \\
C. Mont Royal-Cochem & $30-75^{\circ} \mathrm{NE}-\mathrm{SW}$ & Endertbach-Lineation $140^{\circ} \mathrm{NW}-\mathrm{SE}$ \\
D. Cochem-Moselkern & $0-5^{\circ} \mathrm{N}-\mathrm{S}$ & Elzbach-Lineation $130^{\circ} \mathrm{NW}-\mathrm{SE}$ \\
E. Moselkern-Koblenz & $130-160^{\circ} \mathrm{NW}-\mathrm{SE}$ & \\
\hline
\end{tabular}




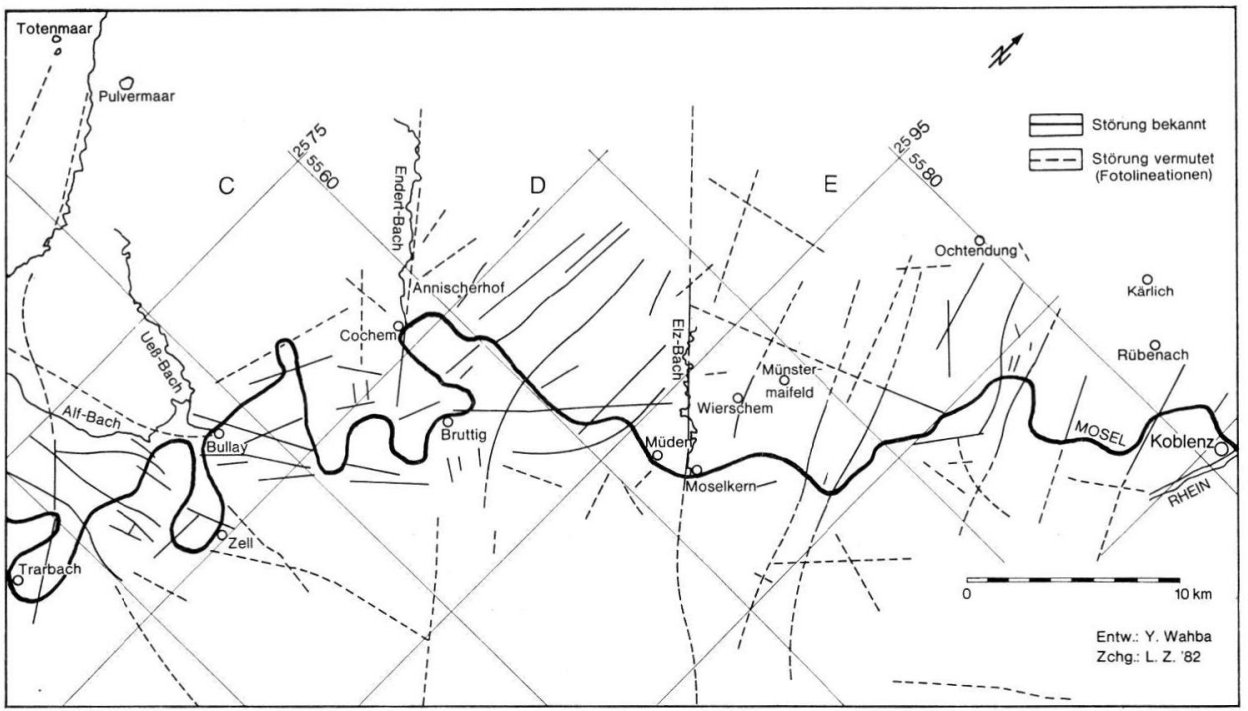

Abb. 1: Tektonische Übersicht des Untermoselgebietes.

Die Schollengliederung basiert auf der Lage und der Richtung der Bruchstrukturen. Die Auswertung von LANDSAT-II-Aufnahmen weist im untersuchten Gebiet fünf, durch langgestreckte Querstörungen (130-150\%) begrenzte Großschollen aus, innerhalb derer Fotolineationen weitgehend einheitliche Streichrichtungen haben.

Diese Großschollen sind von SW nach NE (vgl. Abb. 1):

Die Lineationen zwischen den Großschollen werden aufgrund ihres Charakters als Störungen älterer Anlage, die später z. T. reaktiviert worden sind, gedeutet.

Größere Nebenflüsse der Mosel (Lieser, Alf, Endertbach, Elzbach) sowie wichtige Vulkanlinien der Eifel (Dauner Maare, Mosenberg usw.) folgen teilweise den Großschollengrenzen.

Die folgenden Ausführungen konzentrieren sich auf die Großschollen D und E, da in ihrem Bereich die deutlichsten Indizien für posthume Verstellungen von Terrassen auftreten (vgl. das „Cochemer Gewölbe“, NEGENDANK 1978).

Großscholle D (Cochem - Moselkern): Hier erreicht die Höhenterrasse (HöT) ${ }^{1}$ ) ihre höchste Lage (Annischerhof, 330-334 m), um dann an N-S streichenden Störungen

1) Die Korrelierung der Terrassen auf der „Untermoselscholle“ (E) und der „Mittelmoselscholle“ (A-D) ist noch umstritten. Wir verweisen hier auf die Diskussion einer in Vorbereitung stehenden Arbeit von Negendank. Für die Fragestellung der vorliegenden Arbeit ist es letztlich unbedeutend, ob der Verstellungsbetrag der pleistozänen Hauptterrassen $60-75 \mathrm{~m}$ oder weniger beträgt. Loennertz (1982) glaubt, die Hauptterrassentreppe des Mittelrheins nach Bibus \& SemMEL (1977) an der Mittelmosel exakt wiederzufinden, allerdings in $40-45 \mathrm{~m}$ höherer Position. Auch die Frage, wann der Hauptbetrag der Verstellung der Tertiärbasis erfolgte, ist hier zweitrangig. Für die in Abb. 1 übernommene Terrassenparallelisierung spricht allerdings, daß im Profil Kärlich die paläomagnetische Matuyama/Brunhes-Grenze $(0,7 \mathrm{ma})$ in Moselsedimenten zwischen 175 und $180 \mathrm{~m}$ NN liegt (BrunNacker et al. 1976; BrunNaCKER et al. 1969). Die uHT der Saar, die auf die uHT der Mittelmoselscholle ausläuft, ist normal magnetisiert und gehört der Brunhes-Epoche an (ZöLlER, i. V.). Die Matuyama/Brunhes-Grenze dürfte auf der Mittelmoselscholle in der mHT oder höher zu erwarten sein. 
allmählich nach E auf $310 \mathrm{~m}$ abzusinken ${ }^{2}$ ). Denselben Bewegungssinn zeigt die Tertiärbasis („Vallendar-Stufe"), jedoch mit wesentlich größeren Höhendifferenzen (bis $40 \mathrm{~m}$, insgesamt $172 \mathrm{~m})$.

Die Tatsache, daß die alttertiären Vallendar-Schotter (= Altere Quarzschotter, AQS) an den N-S-Störungen dieser Großscholle ihre höchsten Versatzbeträge erreichen, während die Hauptterrassen ${ }^{1}$ ) erst in der nordöstlich anschließenden Großscholle an NW-SE streichenden Störungen in großem Maße absinken, zeigt, daß an der Untermosel eine ältere (jungtertiäre) und eine jüngere (pleistozäne) Tektonik unterschiedliche Auswirkungen hinterlassen haben.

Großscholle E (Moselkern-Koblenz): Die Scholle wird von der Elzbach-Lineation und bei Koblenz vom Neuwieder Becken begrenzt.

In dieser Scholle treten acht - vorwiegend abschiebende - Querstörungen in 135$160^{\circ} \mathrm{NW}-\mathrm{SE}-$ Richtung auf, wobei Richtungen von $150-160^{\circ}$ überwiegen. Damit ist eine Rechtsdrehung um ca. $20^{\circ}$ gegenüber den bisher kartierten Strukturen des Neuwieder Beckens (MEYER 1979) zu beobachten.

Die großen $150-160^{\circ}$ Lineationen sind nur bis zum Süd- bzw. Westrand des Neuwieder Beckens zu verfolgen. Im Becken selbst werden kleinere Lineationen (ca. $135^{\circ}$, NNW-SSE) sichtbar (MEYer 1979). Somit herrschen im Becken andere Strukturen vor als im anschließenden Moselgebiet.

Die uHT sinkt um insgesamt $78 \mathrm{~m}^{1}$ ) (primäres Gefälle nicht substrahiert, es dürfte, der uNT-Basis nach zu urteilen, maximal $16 \mathrm{~m}$ gewesen sein), bezogen auf den Ostrand der nach W angrenzenden Scholle D (Cochem-Moselkern), ab.

Folgende Höhenwertzusammenstellung ${ }^{1}$ ) verdeutlicht, daß die Absenkung der Tertiärbasis im wesentlichen erst im Pleistozän erfolgte:

\begin{tabular}{ccccccc} 
& $\begin{array}{c}\text { I } \\
\text { Müden (D) }\end{array}$ & $\begin{array}{c}\text { II } \\
\text { Wierschem (E) Rübenach (E) }\end{array}$ & I-II & II-III & I-III \\
\hline Tertiärbasis & $202 \mathrm{~m}$ & $190 \mathrm{~m}$ & $139 \mathrm{~m}$ & $12 \mathrm{~m}$ & $51 \mathrm{~m}$ & $63 \mathrm{~m}$ \\
\hline mHT-Basis & $260 \mathrm{~m}$ & $250 \mathrm{~m}$ & $185 \mathrm{~m}$ & $10 \mathrm{~m}$ & $65 \mathrm{~m}$ & $75 \mathrm{~m}$ \\
\hline & Tertiär bis mHT & & $2 \mathrm{~m}$ & $14 \mathrm{~m}$ & $12 \mathrm{~m}$ \\
\hline
\end{tabular}

(Das stärkere Absinken der mHT gegenüber der Tertiärbasis kann unter Umständen auf ein primär stärkeres Gefälle der mHT zurückzuführen sein.)

Es fällt auch auf, daß auf der Scholle D Tertiär- und HöT-Relikte in einigen Fällen von Fotolineationen begrenzt werden, z. B. die Kieseloolith-Terrasse (KiOT) südöstlich Bruttig und westlich Briedern, HöT südöstlich Pommern.

\subsection{Saar-Nahe-Gebiet (L. ZöLleR)}

Beispiel 1: Hauptterrasse der Nahe zwischen Monzingen und Nußbaum (Blatt 6211 Sobernheim).

2) Es ist denkbar, zwei pleistozäne Höhenterrassen anzunehmen, wodurch der Verstellungsbetrag um einen Betrag bis zur Höhe des - nicht genau bekannten - Vertikalabstandes der beiden Höhenterrassen reduziert würde. Das ändert aber nichts an der Tatsache, daß die HöT an keiner anderen Stelle im Moselgebiet die absolute Höhenlage erreicht wie am Annischerhof. 


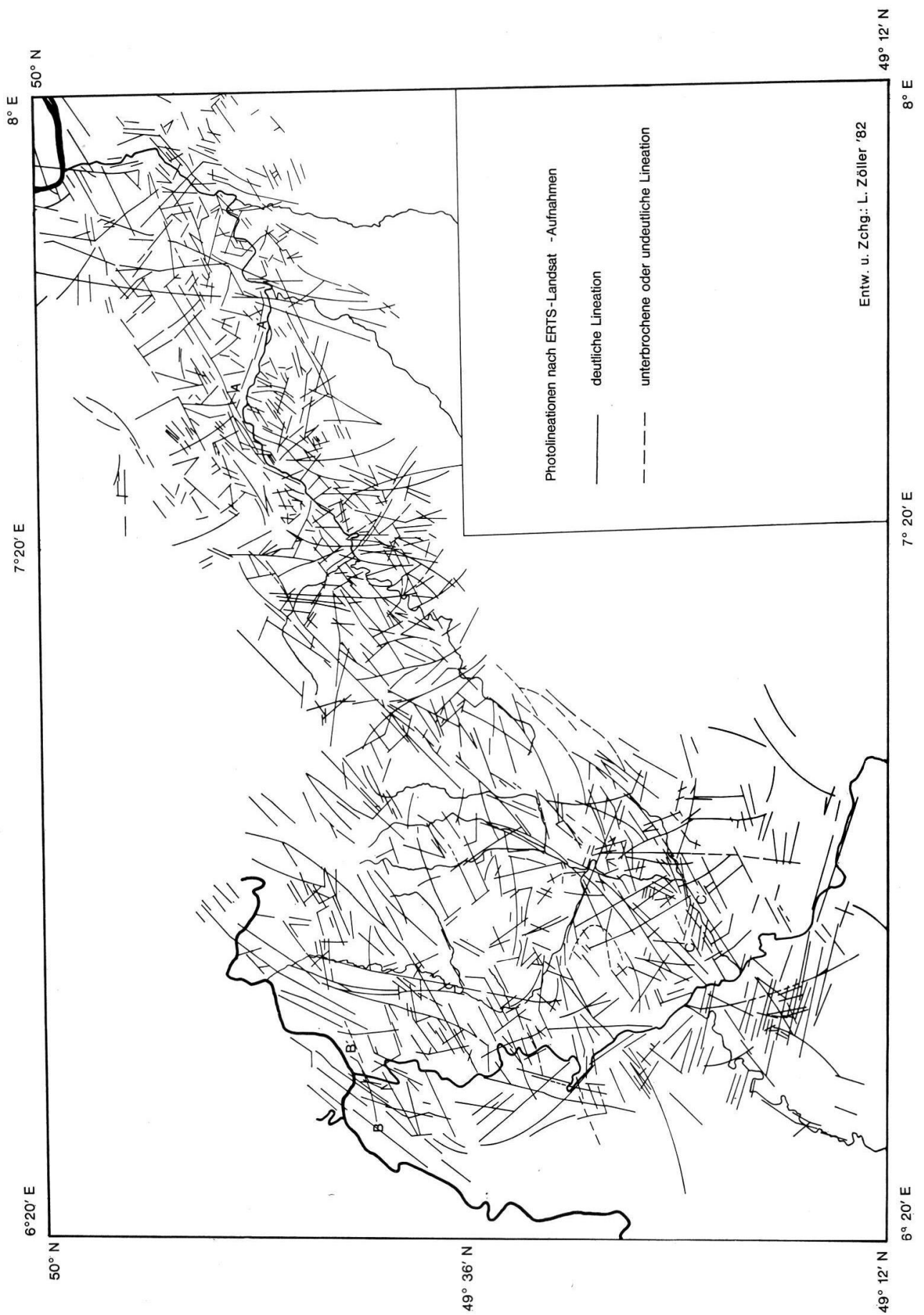

Abb. 2: Saar - Ruwer - Prims - Nahe. Photolineationen nach ERTS-Landsat-Aufnahmen (Kanäle 5, 6, 7, Schwarz-Weiß-Aufnahmen). 
Die WNW-ESE verlaufende Lineation $\left(\mathrm{A}-\mathrm{A}^{\prime}\right.$ in Abb. 2) - Teilabschnitt einer Schar von gleichgerichteten Lineationen parallel zum heutigen Nahelauf - verläuft entlang einer ca. $1 \mathrm{~m}$ hohen Stufe in der Terrassenoberfläche. Beiderseits der Lineation $\mathrm{A}-\mathrm{A}^{\prime}$ wurde die Terrassenbasis in bis zu $9 \mathrm{~m}$ differierender Höhenlage festgestellt, in einem Straßenabschnitt unmittelbar nördlich der Lineation mehrere kleine Abschiebungen von $1-2 \mathrm{dm}$, die die Terrassenbasis über sedimentärem Oberrotliegendem versetzen. Nach ESE $\mathrm{zu}$ verringert sich der maximale Verstellungsbetrag von $9 \mathrm{~m}$ auf $2-3 \mathrm{~m}$ (Sobernheim-Domberg) und schließlich auf $0 \mathrm{~m}$. Im Raum Sobernheim wird eine kräftige postoligozäne Hebung auf den Hunsrück zu auch durch die Höhenlage der Tertiärrelikte belegt. (Näheres in der in Druck befindlichen Arbeit von L. ZöLler, Geomorphologische und quartärgeologische Untersuchungen im Hunsrück-Saar-Nahe-Raum; vgl. BIRKENHAUER 1971.)

\section{Beispiel 2: Niederterrasse (NT) der Saar bei Könen (Blatt 6205 Saarburg)}

Durch umfangreiche Baumaßnahmen zur Saar-Kanalisation und durch refraktionsseismische Messungen stand eine Vielzahl von Daten über Aufbau und Höhenlage der weichselzeitlichen NT zur Verfügung. Daraus geht hervor, daß die NT südlich Könen posthum um ca. $5 \mathrm{~m}$ gehoben wurde. Auch die HT der Saar erreicht in diesem Gebiet ihre größte Höhenlage, sie hat bis zum südlichen Hunsrückrand bei Mettlach insgesamt rückläufiges Gefälle (Zöller i. V.). Die Störung in der NT fällt mit einer WSW-ENEstreichenden Fotolineation ( $B-B^{\prime}$ in $\left.A b b .2\right)$ zusammen, die westlich Könen spitzwinklig von der SSW-NNE-streichenden Ostrandverwerfung der Trierer Bucht abzweigt und das Devon, die Trias und die Talaue der Saar durchsetzt.

Beispiel 3: Terrassen der unteren Prims (Bl. 6606 Saarlouis)

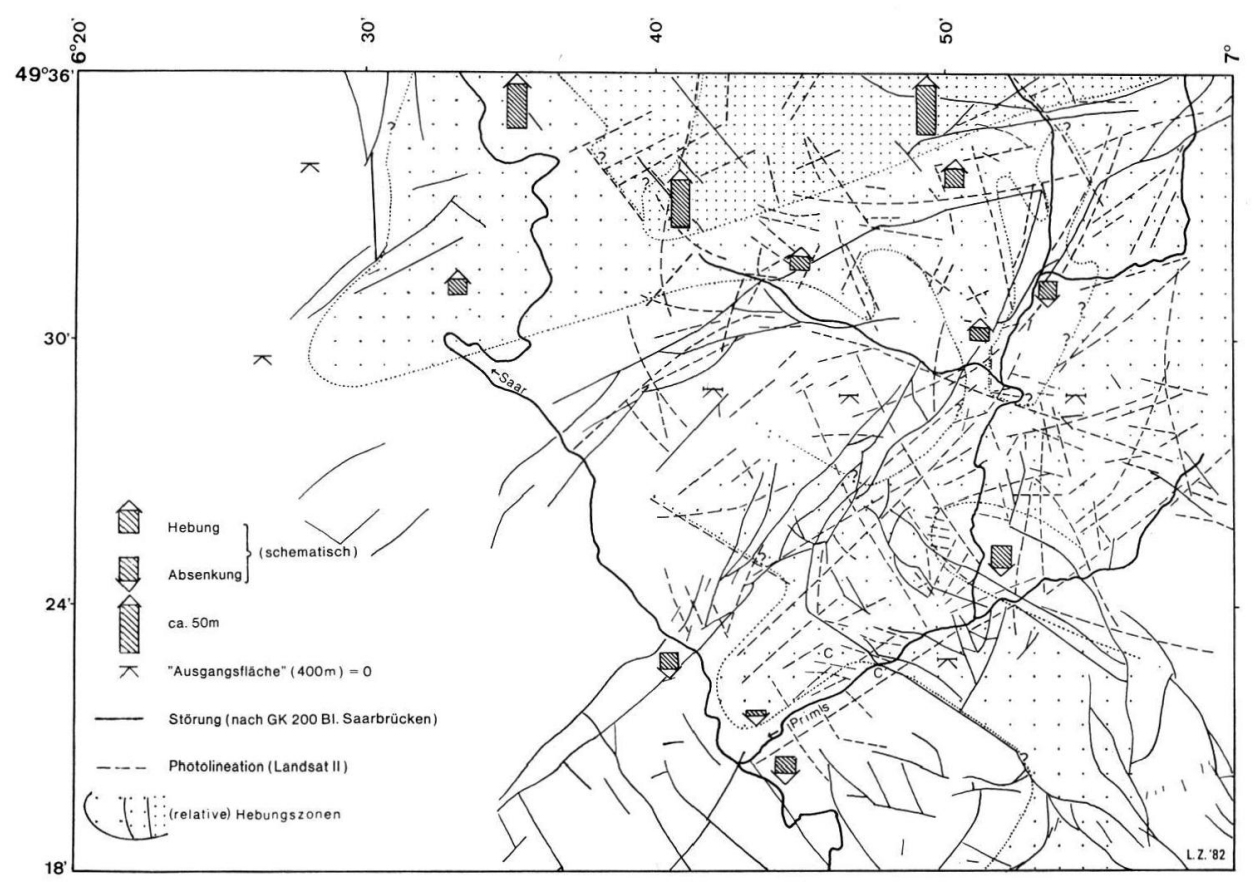

Abb. 3: Tektonische Karte des Prims-Gebietes und jungtertiär-quartäre Vertikalbewegungen. 
Am Westrand des Permokarbon-Horstes von Falscheid (Konzan, MÜller \& KLINKENFiAMMER 1981) gegen den Mittleren Buntsandstein (sm) der Lothringer Senke sind die Terrassen der Prims bei Nalbach verstellt, die NT um ca. $5 \mathrm{~m}$, die HT um ca. $15 \mathrm{~m}$. An diesem Scharnier wurden kurze, auffällige, eng parallelverlaufende Lineationen in WNW-ESE-Richtung kartiert, die gegenüber der NW-SE-verlaufenden Randstörung des Horstes (wie sie in geologischen Karten eingetragen ist) leicht nach links herausdrehen (Lineation $\mathrm{C}-\mathrm{C}^{\prime}$ in $\mathrm{Abb} .2$ und 3). Inzwischen wurde eine bedeutende Störung im sm bei Nalbach in WNW-ESE-Richtung nachgewiesen (KonZAN et al. 1981).

\section{Beispiel 4: Mittlere/obere Nahe}

In den Terrassenlängsprofilen der Nahe zeigt sich ein junges Hebungsgebiet oberhalb der Simmerbachmündung bis Idar-Oberstein ("Querzone“, ZölleR, i. V., s. o.). Es kompensiert nach SW zunehmend den postaquitanen Hebungsbetrag des Hunsrücks gegenüber dem Mainzer Becken und der Kreuznach-Sobernheimer Bucht.

In der gewichteten Fotolineationenkarte (Abb. 4) lassen sich deutlich drei Großschollen mit unterschiedlichem Muster unterscheiden:

1. der Hunsrück mit Vorherrschen SW-NE gerichteter Lineationen, besonders an der Hunsrücksüdrand-Störungszone,

2. die östliche Nahemulde und Teile des Pfälzer Sattelgewölbes, wo die SW-NE gerichteten Strukturen der saalischen „germanotypen Bruchfaltung“ (FALKE 1964) von langgestreckten N-S- bis NNE-SSW-Lineationen durchsetzt werden,

3. die "Querzone" mit charakteristischer Vergitterung SW-NE und NW-SE streichender Lineationen und Lineamente etwa von der Simmerbachmündung ab nach SW. Die stärksten mittel- und jungpleistozänen Terrassenverstellungen (bis $40 \mathrm{~m}$ ) treten im Bereich der Vergitterung der Hunsrück-Südrandzone mit dem E-Rand der „Querzone" auf. Letztere liegt im Bereich der fotogeologisch ermittelten „Zone Nord-Brabant-Schwäbische Alb" (GüNTHER 1977). Unsere Ergebnisse stehen in guter Übereinstimmung mit der von Schwab (1982) belegten Kippung der Nahemulde nach E.

\section{Schlußfolgerung}

Die fotogeologische Schollengliederung erlaubt neben der Bestätigung bereits bekannter regionalgeologischer Einheiten die Ausgliederung weiterer Schollen, die z. T. eine nicht unbedeutende Funktion in der jungtertiär-quartären Tektonik innehatten.

Fotogeologische Methoden können - trotz aller noch offenen Fragen bezüglich dem Abbildungscharakter von Lineationen - ein geeignetes Hilfsmittel zur tektonischen Analyse von Terrassensystemen darstellen, und zwar in zweifacher Hinsicht:

1. Wo Lineationen Terrassenkörper durchsetzen, ermöglichen sie eine gezielte Suche nach posthumen Störungen und stellen somit eine bedeutende Arbeitserleichterung für die Feldarbeiten dar.

2. Durch den analytischen Vergleich der fotogeologischen Schollenmosaike mit quartären und tertiären Bezugshorizonten (Terrassen, Tertiärbasis und -oberkante) können Zonen junger Hebung bzw. Absenkung und Flexuren oder flexurartige Störungszonen räumlich genauer eingegrenzt und flächenhaft dargestellt werden. Voraussetzung bleiben aber auch in diesem Falle eine gründliche Geländearbeit und die Erarbeitung tauglicher Bezugshorizonte. 


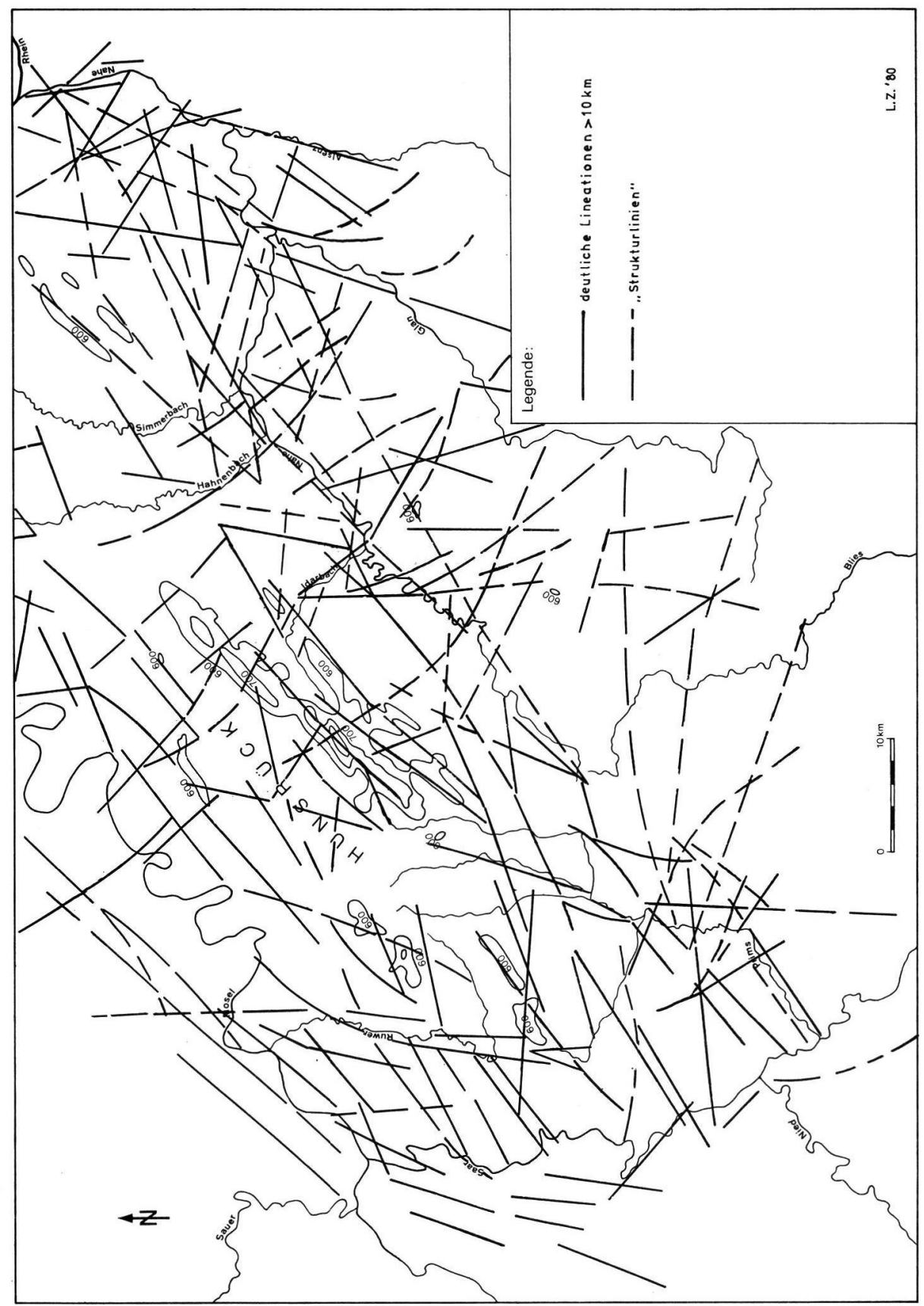

Abb. 4: Saar - Nahe - Hunsrück, Fotolineationen (Landsat II). 


\section{Danksagung}

Die Untersuchungen wurden dankenswerterweise von der "Deutschen Forschungsgemeinschaft" im Rahmen des Schwerpunktprogrammes „Vertikalbewegungen und ihre Ursachen am Beispiel des Rheinischen Schildes" gefördert.

Den Geologischen Landesämtern von Rheinland-Pfalz und des Saarlandes sowie den Straßenbauämtern Trier und Saarbrücken sei für die Genehmigung zur Einsicht in die Bohrarchive herzlich gedankt.

\section{Schriftenverzeichnis}

Ahorner, L. (1962): Untersuchungen zur quartären Bruchtektonik der Niederrheinischen Bucht. Eiszeitalter u. Gegenwart, 13: 24-105, 23 Abb., 7 Tab.; Öhringen.

Bibus, E. \& Semmel, A. (1977): Über die Auswirkungen quartärer Tektonik auf die altpleistozänen Mittelrhein-Terrassen. - Catena, 4: 385-408, 4 Abb., 1 Tab.; Gießen.

Birkenhauer, J. (1971): Zur Talgeschichte des unteren und mittleren Nahegebietes. - Decheniana, 123: 1-18, 2 Abb.; Bonn.

BoENIGK, W. (1978): Die flußgeschichtliche Entwicklung der Niederrheinischen Bucht im Jungtertiär und Altquartär. - Eiszeitalter u. Gegenwart, 28: 1-9, 3 Abb.; Ohringen.

Brunnacker, K., Boenigk, W., Koći, A. \& Tillmans, W. (1976): Die Matuyama/Brunhes-Grenze am Rhein und an der Donau. - N. Jb. Geol. Paläont. Abh., 151: 358-378, 10 Abb., 2 Tab.; Stuttgart.

- , Streit, R. \& Schirmer, W. (1969): Der Aufbau des Quartärprofils von Kärlich/Neuwieder Becken (Mittelrhein). - Mz. Naturwiss. Arch., 8: 102-133, 8 Abb., 3 Tab., Mainz.

FALKE, H. (1964): Zur Tektonik des Rotliegenden im Saar-Nahe-Gebiet. - Jber. u. Mitt. Oberrhein. Geol. Ver. N. F. 46: 35-51, 5 Abb.; Stuttgart.

GÜNTHER, R. (1977): Großfotolineationen des mitteleuropäischen Raumes und ihre geologischtektonische Bedeutung. - Geotekt. Forsch., 53: 42-67, 9 Abb.; Stuttgart.

Konzan, H.-P., Müller, E. \& Klinkhammer, B. (1981): Erläuterungen zur Geologischen Karte des Saarlandes $1: 25$ 000, Blatt Nr. 6606 Saarlouis. - 48 S., 5 Abb., 2 Tab., 6 Taf., 2 Anl.; Saarbrücken (Geol. Landesamt).

Kronberg, H. (1977): Die regionale und überregionale Bruchtektonik Mitteleuropas - Bestandsaufnahmen und Interpretation. - Geotekt. Forsch., 53: 7-41, 21 Abb. im Text u. auf 2 Beil.; Stuttgart.

Loennertz, W. (1982): Die altpleistozänen Terrassen der Mittelmosel. Uberlegungen zur „Horizontalkonstanz" der Terrassen der "Rheinischen Hochscholle“. - Catena, 9: 63-75, 6 Abb., 2 Tab.; Braunschweig.

Meyer, W. (1979): Influence of the Hercynian structures on Cainozoic movements in the Rhenish Massif. - Allg. Vermess.-Nachr., 86:375-377, 1 Abb.; Karlsruhe.

Müller, E., Zöller, L. \& KonZAN, H.-P. (1981): Jungtertiäre und quartäre Tektonik in der NE-Spitze der Merziger Grabenmulde (Saarland). - Eiszeitalter u. Gegenwart, 31: 65-78, 5 Abb.; Hannover.

Negendank, J. F. W. (1978): Zur känozoischen Entwicklung von Eifel und Hunsrück. Sedimentpetrographische Untersuchungen im Moselbereich. - 90 S., 22 Abb., 14 Tab., 6 Ktn., 26 Bilder (= Forsch. dt. Landeskunde 211), Trier (Zentralausschuß für deutsche Landeskunde).

Schwab, K. (1982): Die Hunsrücksüdrandstörung zwischen Birkenfeld und Kirn. - in: Deutsche Forschungsgemeinschaft: Protokoll 6. Koll. Schwerpunkt „Vertikalbewegungen und ihre Ursachen am Beispiel des Rheinischen Schildes“, 3. u. 4. Dezember 1981, Neustadt/Weinstr.: 164-168; Bonn-Bad Godesberg. — [Unveröff.]

$\mathrm{S}$ a telliten a f $\mathrm{n}$ a h men

LANDSAT 1, 15. Mai 76, E - 1392 - 09115, Kanäle 5, 6, 7

LANDSAT 2, 9. März 77, E - 2777 - 09333, Kanäle 5, 67.

Manuskript eingegangen am 30. 7. 1982, Änderungen 2. 11. 1982. 